ICRES 2018: International Conference on Robot

Ethics and Standards, New York, USA, 20-21 August 2018.

https://doi.org/10.13180/icres.2018.20-21.08.p01

\title{
CREATING THE MODERN STANDARD FOR ETHICAL A/IS
}

\author{
JOHN C. HAVENS \\ Executive Director, \\ The IEEE Global Initiative on Ethics of Autonomous and Intelligent Systems, USA
}

\begin{abstract}
There's a phrase among engineers - "You don't build a bridge to fall down." Codes of ethics have existed for decades to help guide roboticists, programmers, and academics in their work to help prioritize safety concerns regarding what they build. But as with the introduction of any new technology, Autonomous and Intelligent Systems (A/IS) have introduced new societal issues engineers must account for in the design and proliferation of their work. Specifically, A/IS deeply affect human emotion, agency and identity (via the sharing of human data) in ways no technology has ever done before. The IEEE Global Initiative on Ethics of Autonomous and Intelligent Systems was created to help address key ethical issues like accountability, transparency, and algorithmic bias in A/IS and to help recommend ideas for potential Standards based on these technologies. The IEEE $\mathrm{P} 7000^{\mathrm{TM}}$ series of standards projects under development represent a unique addition to the collection of over 1300 global IEEE standards and projects. Whereas more traditional standards have a focus on technology interoperability, safety and trade facilitation, the P7000 series address specific issues at the intersection of technological and ethical considerations. Like their technical standards counterparts, the P7000 series empower innovation across borders and enable societal benefit. Standards provide a form of "soft governance" that can be utilized for policy as well as technology design and manufacture. This means that where these (or similar) Standards are being launched by the engineering or technological community it is imperative to have thought leaders from the robotics and A/IS communities join. Along with social scientists and philosophers, these Working Groups also include corporate and policy leaders to best facilitate the discussions on how to move forward on these issues with pragmatic, values-design-driven Standards that can help set the modern definition of innovation in the Algorithmic Age.
\end{abstract}

